\title{
Rapid-rate Non-sustained Ventricular Tachycardias Documentation in Implantable Cardioverter-Defibrillator Predicts the Occurrence of Ventricular Tachyarrhythmia in Patients with Dilated Cardiomyopathy
}

\author{
Wei-Chieh Lee ${ }^{1}$, Masaya Watanabe ${ }^{2}$, Hisashi Yokoshiki ${ }^{3}$, Taro Tenma ${ }^{4}$, Rui Kamada ${ }^{4}$, \\ Masayuki Takahashi ${ }^{4}$, Hikaru Hagiwara ${ }^{4}$, Yumi Takahashi ${ }^{4}$, and Toshihisa Anzai ${ }^{4}$ \\ ${ }^{1}$ Chang Gung Memorial Hospital Kaohsiung Branch \\ ${ }^{2}$ Hokkaido University \\ ${ }^{3}$ Sapporo City General Hospital \\ ${ }^{4}$ Hokkaido University Graduate School of Medicine
}

April 27, 2020

\begin{abstract}
Aims Non-sustained ventricular tachycardia (NSVT) occurs frequently in patients with dilated cardiomyopathy (DCM), especially in high-risk patients. The role of rapid-rate NSVT (RR-NSVT) documented by an implantable cardioverter defibrillator (ICD) in DCM patients has not been fully explored. This study aimed to determine the relationship between RR-NSVT and the occurrence of ventricular tachyarrhythmias (VTAs) in DCM patients with ICD. Methods From December 2000 to December 2017, 136 DCM patients received ICD or cardiac resynchronization therapy defibrillator (CRT-D) implantation for primary or secondary prevention of VTAs. Based on the occurrence of documented RR-NSVT, patients were classified into RR-NSVT (-) or RR-NSVT (+) groups. Result During the median follow-up of 4.5 years, 50.0\% (68/136) of patients experienced [?] 1 episode, and 25.0\% (34/136) of patients experienced [?] 3 episodes of RR-NSVT. Event-free survival for VTAs was significantly higher in the RR-NSVT $(+)$ group whereas those for heart failure admission and cardiovascular mortality were comparable between groups. In the multivariate cox regression analysis, any RR-NSVT showed a positive association with the occurrence of VTAs (HR: 5.087; 95\% CI: 2.374-10.900; p<0.001). In RR-NSVT (+) patients, a cluster ([?] 3 times/6 months) and frequent pattern ([?] 3 runs/day) of RR-NSVT were observed in $42.6 \%$ (29/68) and 30.9\% (21/68) of patients, respectively, who showed further increased incidence of VTAs. Conclusion In DCM patients with ICD/CRT-D, $50.0 \%$ experienced at least 1 episode of RR-NSVT. RR-NSVT documentation showed a positive association with subsequent occurrence of VTAs, suggesting the importance of constructive arrhythmia management for patients with RR-NSVT.
\end{abstract}

\section{Rapid-rate Non-sustained Ventricular Tachycardias Documentation in Implantable Cardioverter- Defibrillator Predicts the Occurrence of Ventricular Tachyarrhythmia in Patients with Dilated Cardiomyopathy}

Wei-Chieh Lee, MD ${ }^{\mathrm{a}, \mathrm{b}, \mathrm{c}}$, Watanabe Masaya, MD, PhD ${ }^{\mathrm{a}}$, Hisashi Yokoshiki, MD, PhD ${ }^{\mathrm{a}, \mathrm{d}}$, Taro Temma, MD, $\mathrm{PhD}^{\mathrm{a}}$, Rui Kamada, MD, $\mathrm{PhD}^{\mathrm{a}}$, Masayuki Takahashi, $\mathrm{MD}, \mathrm{PhD}^{\mathrm{a}, \mathrm{e}}$, Hikaru Hagiwara, MD, PhD ${ }^{\mathrm{a}}$, Yumi Takahashi, $\mathrm{MD}^{\mathrm{a}}$, Toshihisa Anzai, MD, PhD

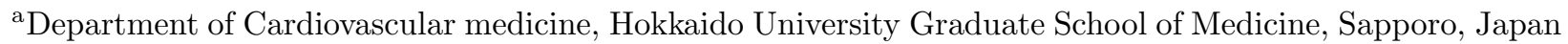

${ }^{\mathrm{b}}$ Division of Cardiology, Department of Internal Medicine, Kaohsiung Chang Gung Memorial Hospital, Chang Gung University College of Medicine, Kaohsiung, Taiwan 
${ }^{\mathrm{c}}$ Institute of Clinical Medicine, College of Medicine, National Cheng Kung University, Tainan, Taiwan

${ }^{\mathrm{d}}$ Department of Cardiovascular Medicine, Sapporo City General Hospital, Japan

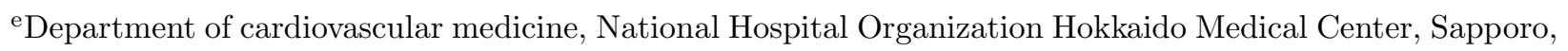
Japan

Address for correspondence:

M. Watanabe, MD, PhD Department of Cardiovascular medicine, Hokkaido University Graduate School of Medicine, Sapporo, Japan

North15, West7, Kita-ku, Sapporo 060-8638, Japan

Tel: $+81-11-706-6973$

Fax: +81-11-706-7874

E-mail: m.watanabe@huhp.hokudai.ac.jp

Running title: Rapid-rate non-sustained ventricular tachycardia and dilated cardiomyopathy

Keywords: Non-sustained ventricular tachycardia; Dilated cardiomyopathy; Recurrent ventricular tachyarrhythmia; A cluster NSVT; A frequent NSVT

\section{ABSTRACT}

Aims

Non-sustained ventricular tachycardia (NSVT) occurs frequently in patients with dilated cardiomyopathy (DCM), especially in high-risk patients. The role of rapid-rate NSVT (RR-NSVT) documented by an implantable cardioverter defibrillator (ICD) in DCM patients has not been fully explored. This study aimed to determine the relationship between RR-NSVT and the occurrence of ventricular tachyarrhythmias (VTAs) in DCM patients with ICD.

\section{Methods}

From December 2000 to December 2017, 136 DCM patients received ICD or cardiac resynchronization therapy defibrillator (CRT-D) implantation for primary or secondary prevention of VTAs. Based on the occurrence of documented RR-NSVT, patients were classified into RR-NSVT (-) or RR-NSVT (+) groups.

\section{Result}

During the median follow-up of 4.5 years, $50.0 \%$ (68/136) of patients experienced [?] 1 episode, and $25.0 \%$ (34/136) of patients experienced [?] 3 episodes of RR-NSVT. Event-free survival for VTAs was significantly higher in the RR-NSVT (+) group whereas those for heart failure admission and cardiovascular mortality were comparable between groups. In the multivariate cox regression analysis, any RR-NSVT showed a positive association with the occurrence of VTAs (HR: 5.087; 95\% CI: 2.374-10.900; p<0.001). In RRNSVT (+) patients, a cluster ([?] 3 times/6 months) and frequent pattern ([?] 3 runs/day) of RR-NSVT were observed in $42.6 \%(29 / 68)$ and $30.9 \%(21 / 68)$ of patients, respectively, who showed further increased incidence of VTAs.

\section{Conclusion}

In DCM patients with ICD/CRT-D, 50.0\% experienced at least 1 episode of RR-NSVT. RR-NSVT documentation showed a positive association with subsequent occurrence of VTAs, suggesting the importance of constructive arrhythmia management for patients with RR-NSVT.

\section{INTRODUCTION}

Non-sustained ventricular tachycardia (NSVT) is defined as more than 3 consecutive beats arising below the atrioventricular node with a rate $>100$ beats $/$ min and lasting less than 30 seconds $^{1}$. In clinical practice, 
NSVT is recorded in a wide range of conditions from apparently healthy, young individuals to patients with significant heart disease ${ }^{2}$. Prior studies suggested that NSVT is a marker for increased risk of subsequent sustained tachyarrhythmias and sudden cardiac death. However, its role in patients with dilated cardiomyopathy (DCM) remains controversial ${ }^{3}$. Zecchin et al. reported that frequent NSVT ([?]3 runs/day) was associated with an increased risk of mortality and ventricular tachyarrhythmias (VTAs), but only in the subgroup of patients with severe LV impairment and idiopathic $\mathrm{DCM}^{4}$. Spezzacatene et al. reported that $38.2 \%$ of DCM patients showed an arrhythmogenic phenotype and that rapid NSVTs ([?] 5 beats, [?] 150 $\mathrm{bpm}$ ) increased the risk of sudden cardiac death ${ }^{5}$. On the other hand, other researchers suggested that NSVT may merely be a surrogate marker of poor left ventricular ejection fraction (LVEF) and electrical instability ${ }^{6}$ or severe $\mathrm{HF}^{7}$ in such patients. Whereas NSVT was generally recorded on ambulatory monitoring (usually 24-hour holter), the prognostic influence of NSVT recorded in defibrillator devices, referred to as rapid rate-NSVT (RR-NSVT), has only been discussed in limited studies ${ }^{8,9}$. Defibrillators can provide the continuous recordings of heart rhythm and, therefore, could be useful to clarify the incidence and clinical impact of NSVT with heart rates above the detection zones.

The current study was sought to explore the incidence of RR-NSVT and its prognostic impacts in DCM patients with defibrillator treatment.

\section{METHODS}

\section{Patient population}

Between December 2000 and December 2017, 170 consecutive DCM patients received an implantation of an implantable cardioverter-defibrillator (ICD) or cardiac resynchronization therapy with defibrillator (CRT-D) for primary or secondary prevention of VTAs in Hokkaido University Hospital. Patients were diagnosed as DCM when they satisfied the following criteria: (1) absence of significant coronary stenosis, (2) abnormal LV function (reduced LVEF $<50 \%$ and dilation), and (3) exclusion of other cardiac and systemic disorders. After exclusion of patients with short follow-up/lost to follow-up, no follow-up echocardiographic data, and no follow-up record of ICD/CRT-D cases, 136 DCM patients were recruited in this study. According to the presence or absence of RR-NSVT, 68 patients without RR-NSVT were classified as the RR-NSVT (-) group and 68 patients with RR-NSVT as the RR-NSVT (+) group.

\section{Device programming}

The ventricular fibrillation (VF) zone detected ventricular events faster than 185-200 beats/min and initial therapy was [?]30 joules (maximum energy of the device). The ventricular tachycardia (VT) zone detected ventricular events faster than 150-170 beats/min, and 3 sequences of ATP were initially attempted. If the arrhythmia continued, the first shock ranged from 10-20 joules and subsequent shocks with maximal energy were delivered until its termination. In cases of patients with documented slow VT, a detection zone lower than 150 beats/min was sometimes programmed. The device-related detection algorithms, such as dual chamber sensing, and the stability and sudden-onset criteria were usually employed for the discrimination of supraventricular tachycardia ${ }^{10}$. The morphology-based algorithm for detecting ventricular tachycardia was used as a nominal setting ${ }^{11}$. The ICD devices were manufactured by Boston Scientific (Marlborough, Massachusetts, USA), Medtronic (Minneapolis, Minnesota, USA), or Abbott/St. Jude Medical (Lake Bluff, Illinois, USA).

\section{Echocardiography}

Echocardiography was performed within one month before ICD/CRT-D implantation and during the followup period in all the study patients. Echocardiographic parameters, including LVEF, LA dimension, LV volume, and estimated pulmonary artery systolic pressure (PASP), were measured using GE Vivid 9 or Philips IE33. LVEF and LV volume (end-diastolic phase and end-systolic phase) were quantified by the two-dimensional guided biplane Simpson's method of disc measurements by echocardiography. PASP was estimated from the tricuspid regurgitant jet velocity using the modified Bernoulli equation and adding the estimated right atrial pressure based on the diameter and collapsibility of the inferior vena cava. 


\section{Follow-up and device-related data collection}

After implantation, all patients were followed up in the clinic at 1 month and every 3-6 months thereafter. The ICD/CRT-D data was reviewed at each follow-up. The diagnostic classification of ICD therapy events was made by electrophysiological specialists according to the stored intracardiac electrograms.

\section{Ethics statement}

The study conforms to the ethical guidelines of the 1975 Declaration of Helsinki and was approved by Institutional Review Committee for Human Research.

\section{Definition}

VTA was defined as a sustained ventricular tachycardia and fibrillation which was treated by ICD and/or lasted $>30$ seconds. RR-NSVT was defined as an episode of VT that met the ICD therapy detection criteria which terminated before defibrillator intervention. A frequent RR-NSVT was defined as [?]3 runs for one day observed before or without following VTAs. A cluster RR-NSVT was defined as [?]3 episodes for 6 months observed before or without following VTAs (Supplemental Figure 1). An electrical storm was defined as [?]3 episodes of VTAs within 24 hours. A CRT responder was defined as a decrease in LV end-systolic volume of [?]15\% and/or an absolute increase of $>5 \%$ in LVEF at the 6-month or 1-year visit compared to the baseline echocardiography ${ }^{12}$.

Cardiovascular mortality was defined as death due to HF, sudden cardiac death, or VTAs. All-cause mortality was defined as death due to cardiovascular mortality, sepsis, or malignancy.

\section{Study endpoints}

The primary endpoint was defined as the occurrence of VTAs. The secondary endpoints were defined as the occurrence of an electrical storm, admission for HF, cardiovascular mortality, and all-cause mortality.

\section{Statistical analysis}

The data are presented as a number (percentage) and mean \pm standard deviation. Clinical characteristics between the study groups were compared by a t-test for continuous variables and chi-squared test for categorical variables. The first occurrence of RR-NSVT during the follow-up period was considered as a time-dependent covariate. For cluster RR-NSVT, the time interval until the occurrence of the third RR-NSVT in the first cluster episode was taken into account. The first appropriate ICD therapy was credited to the (cluster or frequent) RR-NSVT only if it occurred after the first episode to determine whether each form of RR-NSVT was associated with the following VTAs. Cox regression analyses were performed to identify associations with the occurrence of VTAs and were expressed as hazard ratios (HRs) and 95\% confidence intervals (CIs). In the multivariate models, adjustment was performed for significant outcome confounders identified by stepwise selection from the baseline variables with the model entry significance limit set at 0.100 . The cumulative incidence of VTA occurrence and admission for HF and cardiovascular mortality was estimated using the Kaplan-Meier method and compared using a log-rank test between groups. All statistical analyses were performed using statistical software (SPSS for Windows, version 22). A two-sided p-value [?]0.05 was considered significant.

\section{RESULTS}

\section{Baseline characteristics of the study patients}

The baseline characteristics of the study participants are listed in Table 1. The prevalence of CRT-D implantation was significantly higher in the RR-NSVT (-) group (55.9\% vs. $32.4 \%$; $\mathrm{p}=0.009)$. More frequent use of amiodarone (57.4\% vs. $36.8 \% ; \mathrm{p}=0.025)$ and less frequent use of angiotensin-converting enzyme inhibitor/angiotensin II receptor blocker (75.0\% vs. 89.7\%; $\mathrm{p}=0.041)$ was noted in the RR-NSVT (-) group.

Echocardiographic characteristics of the study patients 
Regarding the echocardiographic parameters, LV volume, LVEF and prevalence of moderate to severe mitral regurgitation did not show a significant difference between groups both at the time of implantation and the most recent examination (Table 2). Prevalence of the patients with improved LVEF [?]5\% or $10 \%$ or a decreased LV end-diastolic volume did not differ between groups.

\section{Incidence of RR-NSVT}

During the median follow up of 4.5 years (3.8-5.3 years), 68 (50.0\%) out of 136 patients experienced at least 1 episode of RR-NSVT, and 34 patients (25.0\%) experienced more than 3 episodes (Figure 1A). Of importance, whereas the accumulative incidence of [?]1 episode of RR-NSVT increased in a time-dependent manner, [?]2, [?]3 and [?]4 episodes of RR-NSVT showed a gradual increase before reaching a plateau. A similar tendency was observed in patients with primary (Figure 1B) and secondary prevention (Figure 1C).

In the RR-NSVT (+) group, cluster ([?] 3 times/6 months) and frequent ([?]3 runs/day) RR-NSVT were noted in $42.6 \%(29 / 68)$ and $30.9 \%(21 / 68)$ of patients, respectively.

\section{Clinical outcomes between RR-NSVT (-) and RR-NSVT (+) patients}

Table 3 shows the clinical outcomes in both groups. A higher incidence of VTAs $(11.8 \%$ vs. $67.6 \% ; \mathrm{p}<0.001)$, electrical storm ( $1.5 \%$ vs. $14.7 \%$, $\mathrm{p}=0.009)$, and admission for HF (33.8\% vs. $52.9 \%$; $\mathrm{p}=0.037$ ) were noted in the RR-NSVT (+) group compared to the RR-NSVT (-) group. The incidence of cardiovascular and allcause mortality did not show a significant difference between groups. A longer follow-up period was noted in the RR-NSVT (+) group.

A Kaplan-Meier curve on the occurrence of VTAs showed a significant difference between the groups (logrank $\mathrm{p}<0.001$ ) (Figure 2A), which was consistent in patients with primary (Figure 2B) and secondary prevention (Figure 2C). No significant differences in the event-free survival of HF admission (Figure 3A) and cardiovascular mortality (Figure 3B) were observed between groups.

\section{Risk of ventricular tachyarrhythmias}

Table 4 shows the association between clinical variates and VTA occurrence. On multivariate analyses, male gender (HR: 1.769; 95\% CI: 1.004-3.117; $\mathrm{p}=0.048$ ) and any RR-NSVT (HR: 5.087; 95\% CI: 2.374-10.900; $\mathrm{p}<0.001)$ were found to be independently associated with the occurrence of VTAs.

\section{Influence of different RR-NSVT patterns on VTAs occurrence}

The influence of different RR-NSVT patterns on subsequent VTA occurrence was analyzed within the RRNSVT (+) group. Table 4 shows the adjusted hazard ratio of various RR-NSVT patterns. Frequent, cluster RR-NSVT, and polymorphic RR-NSVTs were examined as the covariates possibly associated with VTA occurrence. Frequent RR-NSVT (HR: 2.503; 95\% CI: 1.317-4.757; $\mathrm{p}=0.005$ ) presented a positive association with VTA occurrence and cluster RR-NSVT (HR: 1.810; 95\% CI: 0.984-3.329; p=0.056) presented a nonsignificant trend of association with VTA occurrence (Table 5). Polymorphic RR-NSVT showed no significant association with VTA occurrence. The time interval (median) from the first occurrence of frequent and cluster RR-NSVTs until the subsequent VTA event were 0 (0-1) and 105 (62-119) days, respectively. Most patients [57.1\% (12/21)] experienced frequent RR-NSVT and subsequent VTAs on the same day. A Kaplan-Meier curve of VTA occurrence showed a significant difference among patients without RR-NSVT and those with RR-NSVT with or without cluster pattern $(\mathrm{p}<0.001)$ (Figure 4). Event-free survival was better in the RRNSVT (-) group compared with the cluster RR-NSVT (-) group $(\mathrm{p}<0.001)$. Between the cluster RR-NSVT $(-)$ and cluster RR-NSVT (+) groups, the log-rank p-value of the Kaplan-Meier curve was 0.050.

\section{DISCUSSION}

The followings are the major findings of the present study. Firstly, half of DCM patients with ICD/CRT-D had at least one episode of RR-NSVT whether they were on primary or secondary prevention. In addition, $25 \%$ had experienced more than 3 RR-NSVT episodes. Secondly, any RR-NSVT had a positive association 
with VTA occurrence. Finally, cluster ([?]3 times/6 months) and frequent patterns ([?]3 runs/day) of RRNSVT were observed in $42.6 \%(29 / 68)$ and $30.9 \%(21 / 68)$ of patients with RR-NSVT, respectively and these patients showed a further increased incidence of VTAs.

\section{Clinical impact of NSVT in DCM patients}

DCM consists of a heterogeneous group of diseases and affects the myocardium without the presence of any significant coronary artery disease ${ }^{13}$. The incidence of NSVT in DCM patients has been reported to widely range between $43 \%$ and $66.5 \%{ }^{4,7}$, and the role of NSVT in DCM remains controversial. Whereas some prior studies suggested that NSVT is a marker for the increased risk of subsequent sustained tachyarrhythmias and SCD ${ }^{3}$, others described NSVT as a potential surrogate marker of poor LVEF and electrical instability ${ }^{6}$ or severe HF ${ }^{7}$ in DCM patients. In our study, $50.0 \%$ of study patients experienced [?] 1 episode of RR-NSVT and any NSVT showed a positive association with VTA occurrence. Of note, there was no significant difference in the incidence of HF symptoms and BNP levels between patients with and without RRNSVT. In addition, the accumulative incidence of cardiac death and heart failure admission was comparable between groups. Recently, Spezzacatene et al. reported that $38.2 \%$ of DCM patients meet the criteria of an arrhythmogenic phenotype defined by the presence of [?]1 of the following: rapid NSVT ([?]5 beats, [?]150 bpm), [?]1000 PVCs/day, [?]50 couplets/day, and syncope and that NSVT and VTAs may occur without signs and symptoms of overt HF and may not be related to the severity of LV dysfunction ${ }^{5}$. Furthermore, they described that a DCM arrhythmogenic phenotype and family history of sudden cardiac death (SCD)/VTAs are predictors for the occurrence of SCD/VTAs. As most patients with RR-NSVT defined in our study corresponds with the definition of an arrrhythmogenic phenotype, our findings may highlight the clinical impact of such phenotypes in DCM patients.

\section{The clinical impact of RR-NSVT in DCM patients}

RR-NSVT, which meets detection criteria but terminates itself before the delivery of defibrillator therapy, is not rare in routine ICD/CRT-D interrogation of DCM patients. Of note, the definition of RR-NSVT is not the same as NSVT episodes identified on a 24-hour holter. RR-NSVT is based on the continuous recording of a patient's heart rhythm and can provide more accurate detection of NSVT above the lowest detection rate. In a study enrolling patients with hypertrophic cardiomyopathy, Wang et al. reported that $38 \%$ of patients without NSVT detected by preimplant Holter had an NSVT detected by ICD during follow-up ${ }^{14}$. To the best of our knowledge, 2 studies examined the clinical influence of RR-NSVT and reported that RR-NSVT was a predictor for future ICD therapy and mortality ${ }^{8,9}$. Chen et al. examined the occurrence of RR-NSVT and its association with ICD shocks and mortality in a SCD-HeFT population, in which ICD therapy was set only for a VF zone [?]188 beats $/ \mathrm{min}^{8}$. Recently, Zhou et al. explored the predictive value of RR-NSVT on appropriate ICD therapy in a real-world setting, in which they set the monitor zone of 140-170 bpm and VT therapy zone above $170-210 \mathrm{bpm}$ as well as the VF zone ${ }^{9}$. In the present study, we focused on DCM patients and evaluated the clinical impact of RR-NSVT in DCM patients with a practical setting of ICD/CRT-D. We demonstrated that any RR-NSVT was associated with a higher incidence of VTAs in both primary and secondary prevention. On the other hand, RR-NSVT did not show a significant association with cardiac mortality. One possible explanation for the difference in influence of RR-NSVT on mortality between prior studies and the current study is the background heart diseases included; prior studies included approximately $50 \%$ of patients with other structural heart disease than non-ischemic cardiomyopathy ${ }^{8,9}$. Further studies are needed to evaluate the clinical influence of RR-NSVT in specific patient populations.

\section{Different patterns of RR-NSVT}

In the current study, half of the study patients experienced [?] 1 episode of RR-NSVT and such incidence increased in a time-dependent matter, whereas those with [?] 3 episodes almost reached a plateau. Currently, few studies evaluated the influence of different NSVT or RR-NSVT patterns that can predict further VTAs. Zecchin et al. reported that frequent NSVT ([?] 3 runs/day) was associated with an increased risk of mortality and VTAs in patients with severe LV dysfunction ${ }^{6}$. Similarly, we identified a higher incidence of VTAs in patients with frequent RR-NSVT compared to those without a frequent pattern despite the presence 
of any RR-NSVT. However, most patients with frequent NSVT $(12 / 21,57.1 \%)$ experienced frequent RRNSVT and subsequent VTA on the same day, which does not provide physicians with ample time to manage antiarrhythmic treatments. On the other hand, cluster NSVTs ([?]3 episodes for 6 months) may serve as a better predictor of the following VTAs as the median time from cluster RR-NSVTs to VTAs was 105 days. Although we found borderline significance in the association between a cluster RR-NSVT and VTAs, this specific pattern of RR-NSVT is an important clue for further treatment during follow-up in such populations, especially in the current era in which home monitoring systems are available. Further studies to evaluate clinical influence of specific NSVT patterns are needed.

\section{Limitations}

We admit some potential limitations in the current study. The first limitation was that the period of patient enrollment was long, and it was a single center study. The second limitation was the relatively small population. The third limitation was inconsistencies in the detection of RR-NSVT as heterogeneous brands of ICD/CRT-D were used. Nonetheless, in our study, RR-NSVT documentation in ICD/CRT-D was important for the prediction of future sustained VTA occurrence in DCM patients. This finding calls for a large registry to detect patients with cluster or frequent RR-NSVTs and analyze treatment and preventive strategies for future VTAs in a similar population.

\section{Conclusions}

In DCM patients with ICD/CRT-D, half of the patients experienced at least 1 episode of RR-NSVT. Any RR-NSVT showed a positive association with VTA occurrence. Constructive arrhythmia management may be desired for patients with RR-NSVT.

\section{Sources of funding}

Doctor Wei-Chieh Lee was supported by the Asia Pacific Heart Rhythm Society for clinical electrophysiology research at the Hokkaido University Hospital.

\section{Disclosures}

None

\section{Conflicts of interest}

All authors declare that they have no conflicts of interest.

\section{Human rights statements and informed consent}

All procedures followed were in accordance with the ethical standards of the responsible committee on human experimentation (institutional and national) and with the Helsinki Declaration of 1964 and later revisions.

\section{REFERENCES}

1. Katritsis D.G., Camm A.J. Nonsustained ventricular tachycardia: where do we stand? Eur Heart J. 2004;25:1093-1099.

2. Marine JE. Nonsustained Ventricular Tachycardia in the Normal Heart: Risk Stratification and Management. Card Electrophysiol Clin. 2016;8(3):525-543.

3. Katritsis DG, Zareba W, Camm AJ. Nonsustained ventricular tachycardia. J Am Coll Cardiol. 2012;13;60(20):1993-2004.

4. Zecchin M, Di Lenarda A, Gregori D, Moretti M, Driussi M, Aleksova A, Chersevani D, Sabbadini G, Sinagra G. Prognostic role of non-sustained ventricular tachycardia in a large cohort of patients with idiopathic dilated cardiomyopathy. Ital Heart J. 2005;6(9):721-727.

5. Spezzacatene A, Sinagra G, Merlo M, Barbati G, Graw SL, Brun F, Slavov D, Di Lenarda A, Salcedo EE, Towbin JA, Saffitz JE, Marcus FI, Zareba W, Taylor MR, Mestroni L; Familial Cardiomyopathy Registry. Arrhythmogenic Phenotype in Dilated Cardiomyopathy: Natural History and Predictors of Life-Threatening Arrhythmias. J Am Heart Assoc. 2015;16;4(10):e002149. 
6. Zecchin M, Di Lenarda A, Gregori D, Merlo M, Pivetta A, Vitrella G, Sabbadini G, Mestroni L, Sinagra G. Are nonsustained ventricular tachycardias predictive of major arrhythmias in patients with dilated cardiomyopathy on optimal medical treatment? Pacing Clin Electrophysiol. 2008;31(3):290-299.

7. Yokoshiki H, Shimizu A, Mitsuhashi T, Furushima H, Sekiguchi Y, Manaka T, Nishii N, Ueyama T, Morita N, Okamura H, Nitta T, Hirao K, Okumura K; Members of the Implantable CardioverterDefibrillator (ICD) Committee of the Japanese Heart Rhythm Society. Prognostic significance of nonsustained ventricular tachycardia in patients receiving cardiac resynchronization therapy for primary prevention: Analysis of the Japan cardiac device treatment registry database. J Arrhythm. 2018;12;34(2):139-147.

8. Chen J, Johnson G, Hellkamp AS, Anderson J, Mark DB, Lee KL, Bardy GH, Poole JE. Rapidrate nonsustained ventricular tachycardia found on implantable cardioverter-defibrillator interrogation: relationship to outcomes in the SCD-HeFT (Sudden Cardiac Death in Heart Failure Trial). J Am Coll Cardiol. 2013; 28;61(21):2161-2168.

9. Zhou Y, Zhao S, Chen K, Hua W, Su Y, Chen S, Liang Z, Xu W, Zhang S. Predictive value of rapidrate non-sustained ventricular tachycardia in the occurrence of appropriate implantable cardioverterdefibrillator therapy. J Interv Card Electrophysiol. 2019 May 9. doi: 10.1007/s10840-019-00557-4.

10. Swerdlow CD. Supraventricular tachycardia-ventricular tachycardia discrimination algorithms in implantable cardioverter defibrillators: state-of-the-art review. J Cardiovasc Electrophysiol. 2001; 12(5):60612.

11. Swerdlow CD, Brown ML, Lurie K, Zhang J, Wood NM, Olson WH, Gillberg JM. Discrimination of ventricular tachycardia from supraventricular tachycardia by a downloaded wavelet-transform morphology algorithm: a paradigm for development of implantable cardioverter defibrillator detection algorithms. J Cardiovasc Electrophysiol. 2002; 13(5):432-41.

12. Park MY, Altman RK, Orencole M, Kumar P, Parks KA, Heist KE, Singh JP, Picard MH. Characteristics of Responders to Cardiac Resynchronization Therapy: The Impact of Echocardiographic Left Ventricular Volume. Clin Cardiol. 2012;35(12):777-780.

13. Wu AH. Management of patients with non-ischaemic cardiomyopathy. Heart. 2007;93(3):403-408.

14. Wang W, Lian Z, Rowin EJ, Maron BJ, Maron MS, Link MS. Prognostic Implications of Nonsustained Ventricular Tachycardia in High-Risk Patients With Hypertrophic Cardiomyopathy. Circ Arrhythm Electrophysiol. 2017; 10(3).

\section{FIGURE LEGENDS}

Figure 1

The accumulative number of patients with rapid-rate non-sustained ventricular tachycardia (RR-NSVT) of different groups:

A: Whole study group: 50.0\% (68/136) of patients experienced at least 1 RR-NSVT, $33.8 \%$ (46/136) of patients experienced 2 episodes of RR-NSVT, 25.7\% (35/136) of patients experienced 3 episodes of RR-NSVT, and $25.0 \%(34 / 136)$ of patients experienced more than 3 episodes of RR-NSVT. There was a significant difference between the first and second accumulative incidence $(\mathrm{p}=0.010)$.

B: Primary prevention group: 48.7\% (37/76) of patients experienced at least 1 RR-NSVT, $30.2 \%(23 / 76)$ of patients experienced 2 episodes of RR-NSVT, $19.7 \%$ (15/76) of patients experienced 3 episodes of RRNSVT, and $18.4 \%$ (14/76) of patients experienced more than 3 episodes of RR-NSVT. There was a significant difference between the first and second accumulative incidence $(\mathrm{p}=0.033)$.

C: Secondary prevention group: 51.7\% (31/60) of patients experienced at least 1 RR-NSVT, 38.3\% (23/60) of patients experienced 2 episodes of RR-NSVT, and 33.3\% (20/60) of patients experienced more than 2 episodes of RR-NSVT. There was no significant difference between any accumulative incidence.

Figure 2

A Kaplan-Meier curve of clinical outcomes 
A: A Kaplan-Meier curve on the occurrence of VTAs. There was a significant difference between the RRNSVT (-) and RR-NSVT $(+)$ groups (log-rank p $<0.001)$.

B: Patients of primary prevention. There was a significant difference between the RR-NSVT (-) and RRNSVT $(+)$ groups $($ log-rank $\mathrm{p}<0.001)$.

C: Patients of secondary prevention. There was a significant difference between the RR-NSVT (-) and RR-NSVT $(+)$ groups (log-rank $\mathrm{p}=0.002)$.

Figure 3

A: A Kaplan-Meier curve on the admission for heart failure. There was no significant difference between the RR-NSVT (-) and RR-NSVT (+) groups (log-rank p=0.494)

B: A Kaplan-Meier curve on cardiovascular mortality. There was no significant difference between the RRNSVT (-) and RR-NSVT $(+)$ groups (log-rank p=0.815).

Figure 4

A Kaplan-Meier curve of VTA occurrence between patients without RR-NSVT (RR-NSVT [-]) and those with RR-NSVT with (RR-NSVT [+] and cluster NSVT [+]) or without a cluster NSVT (RR-NSVT [+] and cluster NSVT [-]). Event-free survival was significantly different among the three groups $(\mathrm{p}<0.001)$. Eventfree survival was lower in RR-NSVT (-) compared to both RR-NSVT $(+)$ and cluster NSVT $(-)(\mathrm{p}<0.001)$, and RR-NSVT $(+)$ and cluster NSVT $(+)(\mathrm{p}<0.001)$. The log-rank p value was 0.050 between RR-NSVT $(+)$ and cluster NSVT (-) and between RR-NSVT (+) and cluster NSVT (+).
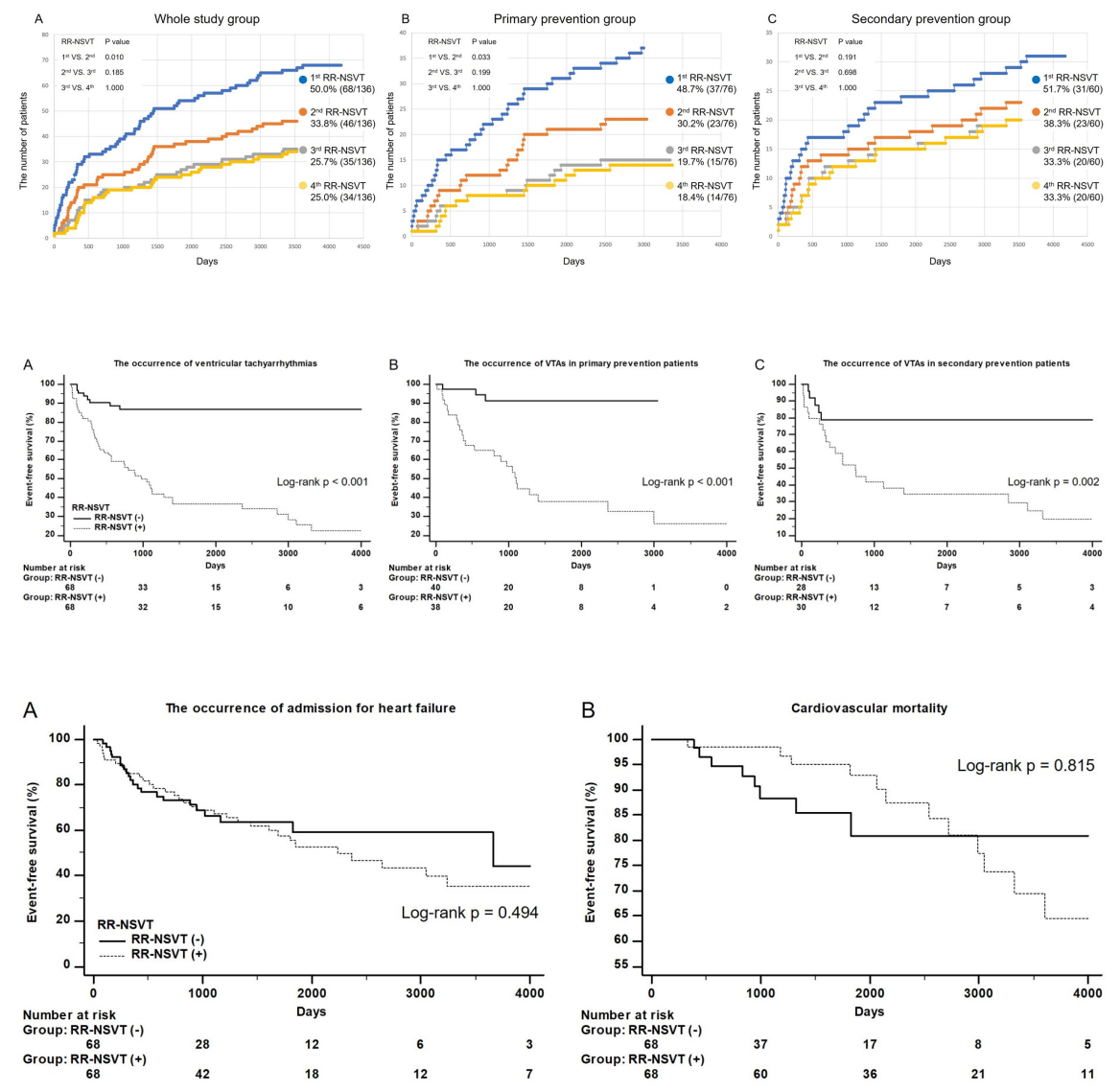
The occurrence of ventricular tachyarrhythmias

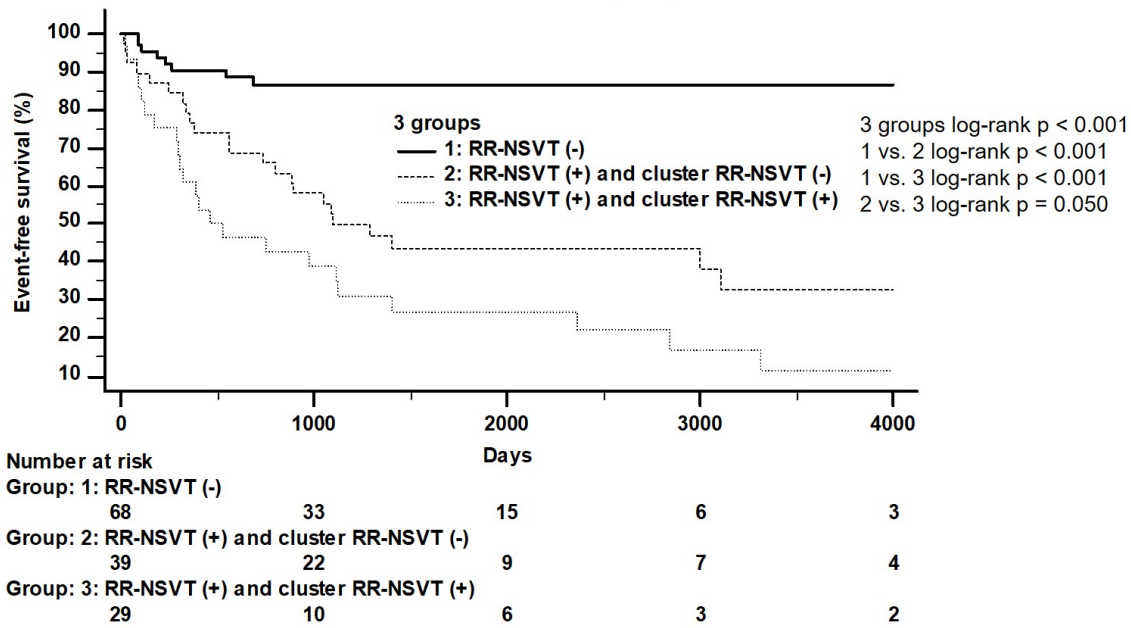

